\title{
Gastrectomy for a patient with early gastric cancer and human immunodeficiency virus (HIV) infection
}

\author{
Kazuhiro Yasuda ${ }^{1}$, Norio Shiraishi ${ }^{1}$, Yosuke Adachi ${ }^{1}$, Seigo Kitano ${ }^{1}$, Hiroshi Kikuchi ${ }^{2}$, Koji Ito $^{3}$, \\ TAKayuki Noguchi ${ }^{3}$, and Kenji Kashima ${ }^{4}$ \\ ${ }^{1}$ First Department of Surgery, Oita Medical University, 1-1 Idaigaoka, Hasama-machi, Oita 879-5593, Japan \\ ${ }^{2}$ Second Department of Internal Medicine Oita Medical University, Oita, Japan \\ ${ }^{3}$ Department of Anesthesiology, Oita Medical University, Oita, Japan \\ ${ }^{4}$ Department of Pathology, Oita Medical University, Oita, Japan
}

\begin{abstract}
Gastric cancer associated with human immunodeficiency virus (HIV) infection is rare, and mostly results in a poor outcome. We report a patient with HIV infection and early gastric cancer successufully treated by gastrectomy. A 49-year-old man with a 6-year history of HIV infection underwent gastric fiberscopy, and a IIc-type depressed lesion was detected in the gastric antrum. With a diagnosis of early gastric cancer, we abided strictly by standard precautions for patients with HIV infection and carried out Billroth I gastrectomy. Histologic examination revealed that the lesion (which measured $0.9 \times$ $0.4 \mathrm{~cm}$ in size), was well differentiated tubular adenocarcinoma confined to the mucosa. A review of the literature disclosed that this is the first reported case of early gastric cancer associated with HIV infection.
\end{abstract}

Key words Gastric cancer · Early gastric cancer - Gastrectomy · Human immunodeficiency virus (HIV) · Acquired immunodeficiency syndrome (AIDS)

\section{Introduction}

The incidence of abdominal surgery in patients with human immunodeficiency virus (HIV) infection is increasing worldwide [1,2]. However, gastrointestinal neoplasms associated with HIV infection are mostly Kaposi's sarcoma or non-Hodgkin's lymphoma, and gastric or colorectal adenocarcinoma is rare [3,4]. Although aggressive and advanced adenocarcinomas of the stomach or colorectum have been reported $[5,6]$, there is no report of early gastric cancer associated with HIV infection.

Recently, we treated a patient with early gastric cancer who had been positive for HIV infection for 6 years. To familiarlize gastroenterologists, surgeons, and pa-

Offprint requests to: $\mathrm{K}$. Yasuda

Received: December 5, 2000 / Accepted: January 26, 2001 thologists, with this condition and operation, we present the clinical, surgical, and pathological data of our patient; we also review the cases in the literature.

\section{Case report}

A 49-year-old man underwent gastric fiberscopy because of a positive result on a fecal occult blood test. He had a 6-year history of HIV infection, which had been controlled with anti-HIV drugs, including indinavir sulfate ethanolate, lamividine, and sanilvudine. On gastric fiberscopy, there was an irregularly shaped, superficially depressed, IIc-type lesion on the anterior wall of the gastric antrum. Histologic examination of the biopsy specimen showed that the lesion was a well differentiated tubular adenocarcinoma of the stomach. Physical examination revealed no palpable lymph nodes, and total colonoscopy disclosed a small adenomatous polyp of the sigmoid colon. Blood data were as follows: red blood cells, $358 \times 10^{4} / \mathrm{mm}^{3}$, white blood cells, $6000 / \mathrm{mm}^{3}$; lymphocytes, $27.5 \%$; $\mathrm{CD}^{4+}$ cell count, $530 / \mathrm{mm}^{3} ; \mathrm{CD}^{4+}$, $31.2 \%$; $\mathrm{CD}^{8+}, 42.2 \%$; $\mathrm{CD}^{4+} / \mathrm{CD}^{8+}, 0.74$; platelets, 16.9 $\times 10^{4} / \mathrm{mm}^{3}$; total protein, $8.10 \mathrm{~g} / \mathrm{dl}$; albumin, $4.58 \mathrm{~g} / \mathrm{dl}$; c-reactive protein (CRP), $0.47 \mathrm{mg} / \mathrm{dl}$; and carcinoembryonic antigen (CEA), $1.3 \mathrm{ng} / \mathrm{ml}$. With a diagnosis of early gastric cancer associated with HIV infection, we carried out Billroth I gastrectomy, on October 16, 2000.

At operation, we abided strictly by standard precautions for patients with HIV infection. All surgeons and the assistant nurse wore surgical caps, masks, goggles, gowns, two pairs of gloves, and stockings (Fig. 1). Surgical instruments were handed from assistant nurse to operator, and the knife, needles, and blood-contaminated gauzes were placed in a tray by the operator and emptied into a needle-stick prevention box (Sharps Container XX-ST08B; Terumo, Tokyo, Japan) by the third assistant (Fig. 2). With the patient under general 
anesthesia with tracheal intubation, distal gastrectomy with limited lymph node dissection (D1 plus no. 7) was performed in the usual manner and Billroth I reconstruction was done using a circular stapler (Proximate ILS 29; Ethicon Endo-Surgery, Cincinnati, OH, USA) and an autosuture stapler (GIA 60; United States Surgi-

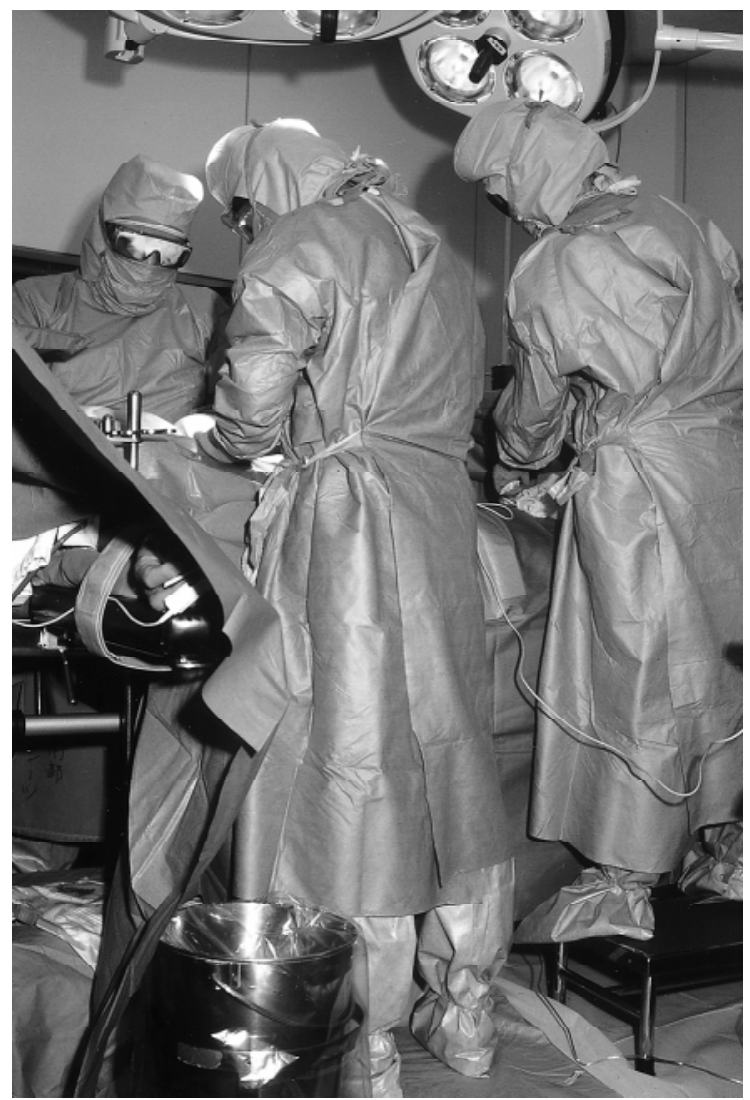

Fig. 1. Surgeons and the assistant nurse wore surgical caps, masks, goggles, gowns, two pairs of gloves, and stockings cal, Norwalk, CT, USA). Abdomional wall closure was done with a continuous blunt-needle polydioxanone (PDS) suture (Ethiguard Needle; Ethicon, Somerville, NJ, USA), and skin closure was done with a skin stapler (Leukoclip SD; Beiersdorf, Hamburg, Germany). The operation time was $2 \mathrm{~h}$ and $20 \mathrm{~min}$, and estimated blood loss was $270 \mathrm{~g}$. The lesion, measuring $0.9 \times 0.4 \mathrm{~cm}$ (Fig. $3 \mathrm{a}$ ), was shown, on histologic examination, to be well differentiated tubular adenocarcinoma confined to the mucosa (Fig. 3b). There was no lymph node metastasis.

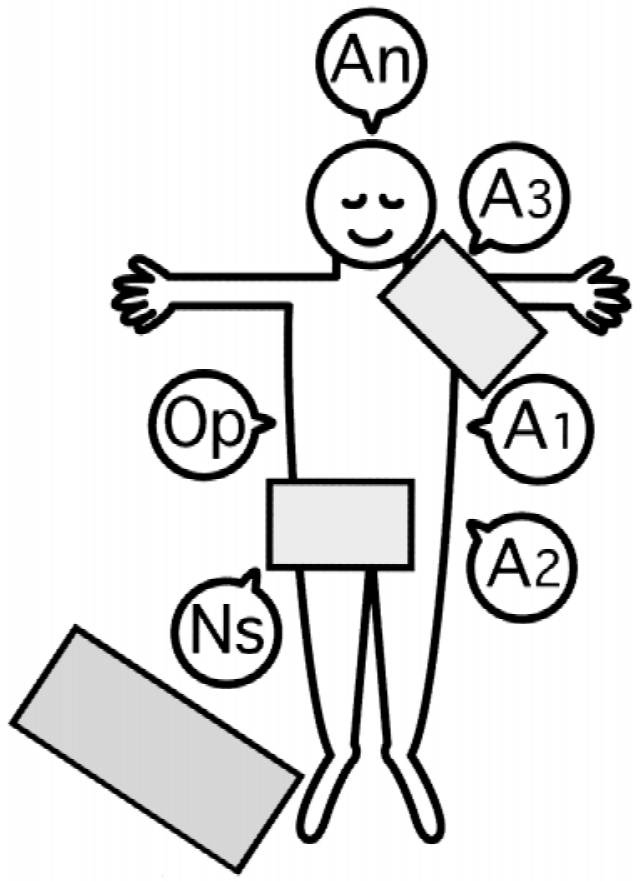

Fig. 2. Knife, needles, and blood-contaminated gauzes were placed in a tray by the operator and emptied into a needlestick prevention box by the third assistant. $O p$, Operator; $A 1$, $A 2, A 3$, assistants; $A n$, anesthetist; Ns, nurse
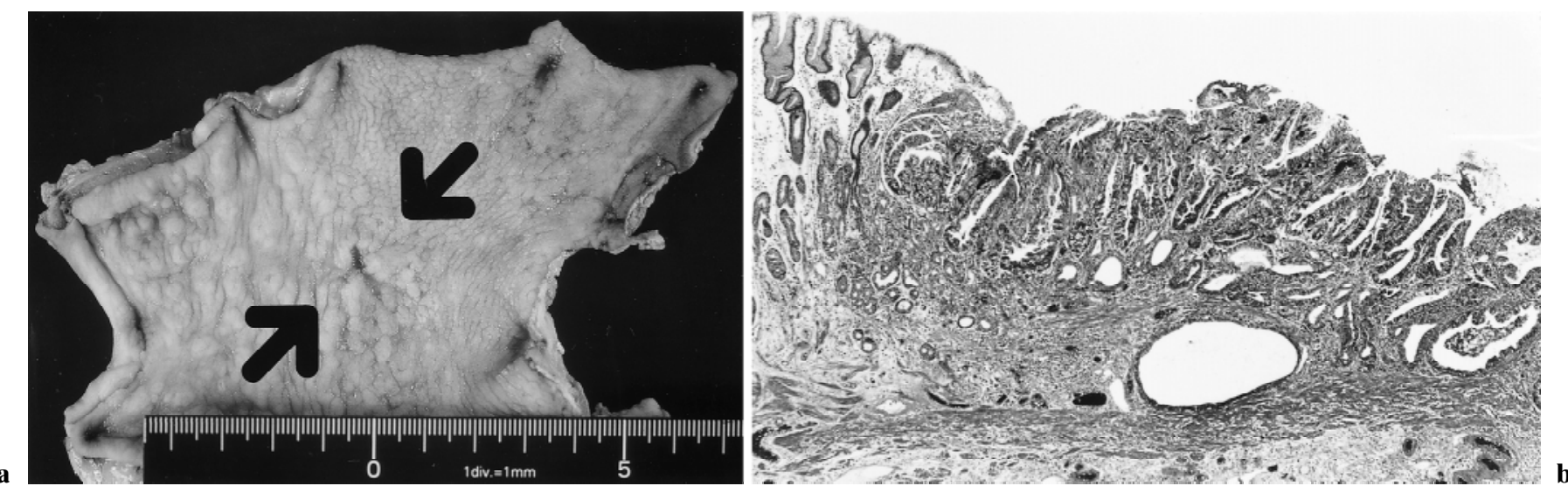

Fig. 3. a There was a superficially depressed, IIc-type lesion, measuring $0.9 \times 0.4 \mathrm{~cm}$, on the anterior wall of the gastric antrum (arrows). b Histologic examination revealed that the lesion in the gastric antrum was well differentiated tubular adenocarcinoma confined to the mucosa. H\&E, $\times 10$ 
The patient recovered uneventfully, and was discharged in good clinical condition on postoperative day 14.

\section{Discussion}

Gastric surgeons are now involved in the treatment of patients with HIV infection or acquired immunodeficiency syndrome (AIDS), because these patients sometimes develop acute abdominal symptoms, opportunistic intraabdominal infections, and gastrointestinal neoplasms. The incidence of abdominal surgery in patients with AIDS was reported to be $2.1 \%-4.2 \%$ in early reports from the United States $[1,7,8]$. Conditions necessitating abdominal surgery were cholecystitis, splenic disorders, acute appendicitis, and peritonitis. Although operations performed because of perforated peptic ulcer or bleeding gastric lymphoma were also described, there are few reports concerning the association of gastric adenocarcinoma and HIV infection [3].

Cappell [3] reported a 36-year-old woman with a highly aggressive gastric adenocarcinoma associated with HIV infection. The patient was admitted with vomiting, weight loss, and a palpable abdominal mass. Roentgenography and endoscopy revealed an ulcerated gastric tumor obstructing the antrum. Distal gastrectomy with Billroth II reconstruction was performed, but peritoneal dissemination developed 1 month after the operation. The resected stomach showed that the tumor was a poorly differentiated, mucin-producing adenocarcinoma containing signet-ring cells and extending down to the serosa.

There are several reports of Japanese patients with AIDS and gastric adenocarcinoma. Ishikawa et al. [9] reported a 39-year-old man with hemophilia A and AIDS who died of disseminated intravascular coagulation (DIC) and multiple organ failure (MOF) caused by bleeding gastric adenocarcinoma. Autopsy revealed that the tumor in the gastric antrum was signet-ring cell carcinoma involving the perigastric, hepatic, paraaortic, and cervical lymph nodes. Uchida et al. [5] reported a 50-year-old man with hemophilia A and AIDS with a tumor in the gastric cardia who presented with dysphagia and weight loss. Endoscopic biopsy showed that the tumor in the gastric cardia was signet-ring cell carcinoma. The patient died of massive tumor bleeding 4 months after the diagnosis.

Several authors suggest the presence of aggressive tumor behavior and young age in patients with gastrointestinal cancers associated with HIV infection $[3,6,9,10]$. Read et al. [10] described a 26-year-old homosexual man with AIDS who presented with Listeria monocytogenes sepsis and small-cell carcinoma of the rectum. At laparotomy, metastases to the liver and lymph nodes were found, and palliative rectal excision was performed. The polypoid tumor, measuring $6 \times$ $4 \mathrm{~cm}$ in size, was small-cell neuroendocrine carcinoma invading the muscular layer and lymphatic channels. The patient died of metastatic recurrences 16 months after the operation, and tumor-related destruction of the rectal mucosa was considered to have facilitated the bloodstream invasion and subsequent sepsis caused by Listeria monocytogenes.

Cappell et al. [6] reported a 36-year-old man, an intravenous drug abuser, who presented with fatigue and weight loss. He had a large mass in the sigmoid colon, occupying the entire pelvis, involving the urinary bladder, and producing bilateral hydronephrosis. One month after admission, a rigid abdomen developed and laparotomy confirmed perforation of the sigmoid colon. They stressed that the patient presented atypically, at a young age, without known risk factors for colon cancer. Immunodeficiency, both of the acquired and congenital types, may be associated with the early development and rapid progression of gastrointestinal cancers [1113].

It was surprising that a small early gastric cancer was found in our patient with HIV infection. He had not complained of any abdominal symptoms, and the fecal occult blood test, followed by gastric fiberscopy, allowed the detection of the early gastric cancer. Although most gastrointestinal malignancies associated with HIV infection or AIDS are characteristic for their highly aggressive behavior, some tumors can be detected at an early stage. Fecal occult blood testing and screening gastric fiberscopy may be important for the successful treatment of gastric cancer in patients with HIV infection.

Elective abdominal surgery for patients with HIV infection is rare in Japan. We followed the operative procedures reported previously for a patient with rectal cancer and HIV infection [4]. Preliminary meetings with the surgeons, nurses, and anesthetists were held, and no operative accident, including needle-stick injury, occurred during our operation. A questionnaire survey of Japanese surgeons in 126 University Hospitals and Institutes showed that approximately $40 \%$ had no manuals for operative procedures in patients with HIV infection, but $70 \%$ stated that they would perform emergency surgery for patients with HIV infection [14]. We hope that this case report will contribute to the further understanding of gastric cancer surgery associated with HIV infection.

Acknowledgments We thank $\mathrm{Mr} \mathrm{H}$. Yoshida for his excellent photography and Miss Y. Kawano for her technical assistance. 


\section{References}

1. LaRaja RD, Rothenberg RE, Odom JW, Mueller SC. The incidence of intra-abdominal surgery in acquired immunodeficiency syndrome: a statistical review of 904 patients. Surgery 1989;105: 175-9.

2. Wilson SE, Robinson G, Williams RA, Stabile B, Cone L, Sarfeh IJ, et al. Acquired immunodeficiency syndrome (AIDS): indications for abdominal surgery, pathology, and outcome. Ann Surg 1989;210:428-34.

3. Cappell MS. A highly aggressive gastric adenocarcinoma associated with human immunodeficiency virus infection (letter). Am J Gastroenterol 1990;85:1199-200.

4. Mori M, Adachi Y, Suehiro T, Sugimachi K, Yoshizawa S, Kashiwagi S, Takahashi S. Operation for rectal cancer in patients with HIV infection (in Japanese). Operation 1990;44:1297-300.

5. Uchida T, Midorikawa J, Takeda H, Shichishima T, Higuchi T, Yui T, et al. Signet cell carcinoma of the stomach in a patient with acquired immunodeficiency syndrome: a case report. Jpn J Clin Oncol 1989;19:75-8.

6. Cappell MS, Yao F, Cho KC. Colonic adenocarcinoma associated with the acquired immunodeficiency syndrome. Cancer 1988;62: 616-9.

7. Barone JE, Gingold BS, Nealon TF, Arvanitis ML. Abdominal pain in patients with acquired immunodeficiency syndrome. Ann Surg 1986;204:619-23
8. Nugent P, O'Connell TX. The surgeon's role in treating acquired immunodeficiency syndrome. Arch Surg 1986;121:111720.

9. Ishikawa M, Suzuki S, Akutsu Y, Toyota T, Nose M, Sakai H, Mori K. An autopsy case of AIDS with hemophilia A who died of DIC and gastrointestinal bleeding associated with gastric carcinoma (signet ring cell carcinoma)(Japanese with English abstract). Jpn J Clin Hematol 1994;35:886-91.

10. Read EJ, Orenstein JM, Chorba TL, Schwartz AM, Simon GL, Lewis JH, Schulof RS. Listeria monocytogenes sepsis and small cell carcinoma of the rectum: an unusual presentation of the acquired immunodeficiency syndrome. Am J Clin Pathol 1985;83: 385-9.

11. Kinlen LJ, Webster AD, Bird AG, Haile R, Peto J, Soothill JF, et al. Prospective study of cancer in patients with hypogammaglobulinemia. Lancet 1985;I:263-6.

12. Scholefield JH, Northover JM, Carr ND. Male homosexuality, HIV infection, and colorectal surgery. Br J Surg 1990;77:4936.

13. Adachi Y, Mori M, Kido A, Shimono R, Suehiro T, Sugimachi K. Multiple colorectal neoplasms in a young adult with hypogammaglobulinemia: report of a case. Dis Colon Rectum 1992;35:197200.

14. Takeda Y, Eriguchi M, Fujii Y. Results of a questionnaire survey concerning surgical operations for HIV-infected patients (in Japanese with English abstract). J Jpn Clin Surg 1995;56:5038. 